\title{
Statistical Measurement of Peptide Nucleic Acid-Assisted Self-Assembly of Gold Nanocluster Dimers
}

\author{
Stephen D. House ${ }^{1}$, Dilhara R. Jayarathna ${ }^{2}$, Catalina Achim², Judith C. Yang ${ }^{1}$ \\ 1. Department of Chemical and Petroleum Engineering, University of Pittsburgh, Pittsburgh, PA (USA) \\ 2. Department of Chemistry, Carnegie Mellon University, Pittsburgh, PA (USA)
}

Hybrid gold nanoparticle (NP)-DNA systems have found use in sensing, imaging, catalysis, therapeutic, diagnostic, and drug delivery applications [1-3]. Au NP conjugates bearing a discrete number of oligonucleotide per particle are particularly useful in bio-diagnostics and as building blocks for selfassembling nanostructures. Synthetic nucleic acid analogues present the potential for expanding the properties that these systems can display. Peptide nucleic acid (PNA) has a higher affinity for DNA than DNA itself and could offer superior selectivity for binding to specific positions on DNA nanostructures. The synthetic routes for modifying the charge and chiral properties of PNA are also relatively simpler than for DNA. Despite being the simplest hybrid system, Au NP dimers have garnered significant interest due to their significant plasmonic coupling enhancement. Most of the NPs used in DNA and PNA functionalization have diameters $>2 \mathrm{~nm}$ (the majority are $>10 \mathrm{~nm}$ ). However, ultra-small $\mathrm{Au}$ nanoclusters (NCs), $<2 \mathrm{~nm}$, possess different properties than their larger kin and are also more biocompatible. The synthesis of Au NC-PNA conjugates combines the desirable properties of PNA and $\mathrm{Au}$ NCs together, paving the way for the construction of new functional hybrid nanomaterials.

We used $\left[\mathrm{Au}_{25}(\text { Captopril })_{18}\right]^{-}($size $\sim 1 \mathrm{~nm}) \mathrm{NCs}$ as the inorganic component and 8- and 10- base pair PNA to create $\mathrm{Au}$ NC-PNA conjugates (Figure 1a). The stoichiometry and purification of the Au-NC PNA single strand conjugates have been based on the use of gel electrophoresis. Their properties in solution have been determined by variable temperature UV-Vis and CD spectroscopy. Confirming and quantitatively characterizing the successful formation of dimers of Au-NCs by the hybridization of $\mathrm{Au}$ NC-PNA single strand conjugates that have complementary sequences was done by S/TEM. Given the $\leq 1 \mathrm{~nm}$ size of the NCs involved, only S/TEM possessed the ability to directly resolve the NCs in sufficiently statistically robust populations.

Here we present an approach we designed for statistically quantifying the degree of self-assembly in the $\mathrm{PNA} / \mathrm{Au}-\mathrm{NC}$ conjugate systems from collections of S/TEM micrographs. Conventional approaches to analyzing spatial distributions of particles tend to assume a random distribution that follows a welldefined function, such as a Poisson distribution. This assumption may not be valid for S/TEM micrographs. First, the micrographs have finite dimensions. NPs located within a certain range of the edges of micrographs have their spatial neighborhoods artificially truncated. The higher the image magnification is, the greater the fraction of peripheral NPs in a given image that are affected, which can significantly skew the measurements. Second, while lower magnifications also help in gathering statistically robust populations of NPs, when imaging such small particles as $\mathrm{Au}_{25} \mathrm{NCs}(<1 \mathrm{~nm})$ there is only so low a magnification one can use before the NCs cannot be clearly resolved. Third, the variation in areal density between micrographs in a given image set also impacts what "random" should be for that set. As the number of NPs in a given area changes, so too does what a random distribution would look like. Since there is no way to avoid multi-image datasets - needed to build up sufficient population sizes at these magnifications - this effect must also be addressed. 
In our approach, we account for these issues by simulating (multiple) image sets using the same image parameters as the experimental micrographs to produce random distributions that are specifically tailored to a specific dataset. These simulations account for the scale, physical dimensions, and areal densities of the NPs of each micrograph in the dataset, and follow the same distribution of particle sizes as the sample. The desired analysis is then applied to each simulated micrograph and combined with the others in the dataset to produce a more accurate "random distribution" that more accurately reflects the reality of the experimental micrographs. These distributions can be directly compared, enabling more meaningful statistical analysis of the samples.

Because the expected self-assembling structures were dimers, a clear deviation from random should be observed in the first nearest neighbor $(1 \mathrm{nn})$ distance. $1 \mathrm{nn}$ distance histograms generated from the experimental and simulated random datasets for the annealed self-complementary sample are overlaid in Figure 1c. The sharp spike in the experimental distribution is due to pairing up of NCs by the hybridization of the PNA single strands. The vertical dashed line identifies the maximum distance that would be expected if the paired PNA single strands were fully extended and is in excellent agreement with the S/TEM observations. The fraction of conjugates that had successfully hybridized to form dimers of $\mathrm{Au} \mathrm{NCs}$ was estimated by comparing the experimental distribution of distances outside of the spike to a fitted simulated distribution of inter Au-NC distances in the case of a random distribution (the difference between the experimental and simulated distributions corresponds to the fraction of Au NCs that are not accounted for by purely random chance). For the sample shown in the Figure, more than $44 \%$ of the NCs had dimerized, a higher fraction than that of the hetero-paired sample (29\%). It is also notably higher than previously reported attempts at DNA or PNA-assisted dimerization, demonstrating the efficacy of our synthetic protocols. A user-friendly GUI-based program has been written to facilitate the process of experimental image analysis, image simulation, and statistical analysis of the resulting distributions. We are currently expanding the approach to systems with larger numbers of PNA tails and will continue to add analysis functionality appropriate to their needs. [4]

\section{References:}

[1] MC Daniel and D Astruc, Chemical Reviews 104 (2004), pp. 293-346.

[2] EC Dreaden, et al., Chemical Society Reviews 41 (2012), pp. 2740-2779.

[3] E Boisselier and D Astruc, Chemical Society Reviews 38 (2009), pp. 1759-1782.

[4] This work was supported by NSF DMREF through contract No CHE-1534630, NSF grant CHE 1413202, and the ETEM Catalysis Consortium (ECC, funded through U.Pitt and Hitachi High Technologies Corp.).
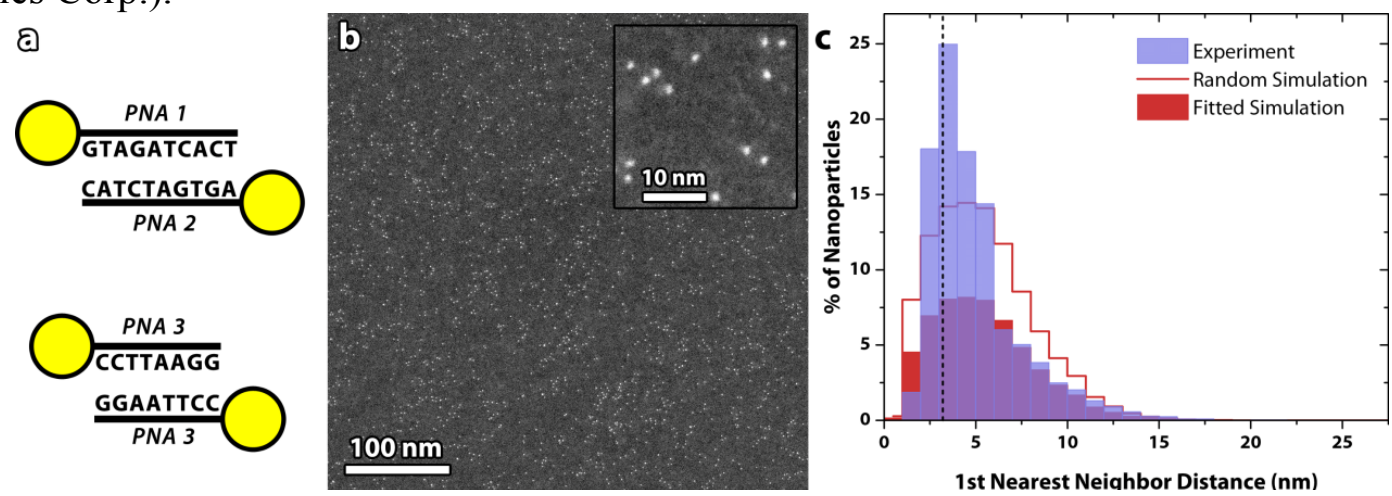

Figure 1. (a) Au NC-PNA conjugates produced in this study. (b) Example ADF-STEM micrograph from the annealed PNA 3 system, and (c) corresponding 1nn distance histograms for the experimental, simulated random, and fitted simulation datasets. 